\title{
ASSOCIATION OF ONCOGENE RAC 1 WITH HER-2 TUMORS AND WITH AGGRESSIVITY OF TRIPLE NEGATIVE TUMORS
}

Maria Amelia Carlos Souto Maior Borba', Joana Darc Rosendo dos Santos', Nancy Cristina Ferraz de Lucena Ferreira', Darley de Lima Ferreira Filho', Glauber Moreira Leitão', Danyelly Bruneska Gondim Martins¹

'Serviço de Mastologia do Hospital Barão de Lucena e Prospecmol, Laboratório de Imunopatologia Keizo Asami, Universidade Federal de Pernambuco - Recife (PE), Brazil.

Objective: To evaluate the expression of RAC 1 in patients with breast cancer and its molecular subtypes. Method: Samples were collected from 41 patients who underwent mastectomy at the Barao Lucena Hospital, 10 luminal A, 15 luminal B, 1 luminal (undifferentiated), 6 HER-2, 9 Triple- negative. RNA was purified by RNAeasy KIT (Qiagen) and quantified by Nano Drop 2000 (Thermo). The cDNA was synthesized with the QuantINova ReverseTranscripition kit (Qiagen) and real-time PCR was performed in StepOnePlus (Applied Biosystems) with the Go Taq Qpcr Master Mix kit (Promega ). The expression of b-actin was used as endogenous control and the ACT was calculated to analyze the reactive quantification of RAC 1 in each sample. Statistical analyzes were performed with R. Results: The relative expression of RAC 1 presented a metric behavior. The HER2 subtype had the highest RAC 1 expression compared to luminal $(\mathrm{p}=0.0006899)$, even when stratified in Luminal A $(\mathrm{p}=0.003592)$ and in Luminal $\mathrm{B}(\mathrm{p}=0.00762)$ and with larger tumor size ( $\mathrm{p}=0.01441)$. No association was observed between RAC 1 expression and KI-67 LOW (KI-67 lower $20 \%$ ), with p values ranging from 0.2186 to 0.9472 . Conclusion: The absence of the estrogen receptor seems to amplify the expression of RAC 1 in response to the metabolic pathway DE her 2. Patients who underwent hysterectomy showed a reduction in the expression of RAC 1 . RAC 1 presents a great potential for new progression studies tumor in triple-negative tumors. 